\title{
Three-Dimensional Simulations and Economical Solutions for Cavitation in Hollow-Jet Dispersive Valves
}

\author{
T. C. L. Xavier and J. P. Ortiz \\ Department of Mechanical Engineering, Polytechnic School, University of São Paulo, Brazil \\ †Corresponding Author Email: jportiz@usp.br
}

(Received October 30, 2020; accepted April 4, 2021)

\begin{abstract}
Cavitation characteristics in Hollow-Jet valves and possible solutions were investigated in the present work. Three-dimensional numerical simulations - CFD (Computational Fluid Dynamics) were carried out in an unsteady state, considering homogenous multiphase flow, to identify the phenomenon in these components. Different turbulence and cavitation models were assessed to reach the best compromise of models. The results confirmed the occurrence of cavities with a mixture of vapor and liquid at the valve tip. The cavities are followed by a vortex generation near the same region. These vortices are the result of a high-velocity gradients, especially in the shear region of the discharge pipe wall, and they are followed by the detachment of the cavities from the valve tip. The methods and models were validated by a reproduction attempt of the results from a similar work on literature. Solutions to avoid or reduce cavitation were proposed and analyzed. Refurbishment and protective coatings against cavitation were particularly described, to envisage an economical solution to reuse the valves, avoiding their disposal or replacement.
\end{abstract}

Keywords: CFD turbulence; Multiphase flow; Hollow-Jet; Dispersive valves; Coating.

\section{NOMENCLATURE}

$\begin{array}{ll}\text { ANA } & \text { Agência Nacional de Águas } \\ \text { CFD } & \text { Computational Fluid Dynamics } \\ \text { d } & \text { valve diameter } \\ \text { FANS } & \text { Favre-Averaged Navier-Stokes } \\ \text { FEMA } & \begin{array}{l}\text { Federal Emergency Management } \\ \text { Agency }\end{array} \\ \text { MANANG } & \begin{array}{l}\text { Maintenance Générale des Ouvrages } \\ \text { D'art }\end{array} \\ \text { PISO } & \text { Pressure-Implicit Split-Operator } \\ \text { RANS } & \text { Reynolds-Averaged Navier-Stokes } \\ \text { SIMA } & \text { Secretaria de Infraestrutura e do Meio } \\ & \text { Ambiente, Governo do ESP, Brasil }\end{array}$

\section{INTRODUCTION}

The use of dispersive valves for energy dissipation is usually for submerged or free-discharge applications. One of the most common problems faced for these components, specifically for the Hollow-Jet valves family, is the cavitation phenomenon. The continuous operation in this condition may jeopardize the whole structure of the component and affect the surrounding environment. According to recent reports produced by USSD (2017), this type of valve has had cavitation problems, and its use is less and less recommended. On the other hand, in many dams around the world,

$\begin{array}{ll}\text { SST } & \text { Shear-Stress Transport } \\ \mathrm{t} & \text { flow time } \\ \text { USACE } & \text { U.S. Army Corps of } \\ & \text { Engineers } \\ \text { USBR } & \text { U.S. Bureau of Reclamation } \\ \text { USSD } & \text { U.S. Society on Dams } \\ \Delta t & \text { time step size } \\ \text { Superscripts } & \text { dimensionless } \\ * & \text { dimensionless measurement } \\ + & \text { of distance from a wall }\end{array}$

these valves are still being used and refurbished. This is the case for the valves of Yellowtail Dam (EUA) (Hydro Review 2009), Palisades Dam (EUA) (USBR 2019), Bissorte Dam (France) (MANANG 2015), Kakki Dam (India) (KakkiAnathodu Dams 2019), and Anderson Ranch Dam (Hydro Review 2012).

In Brazil, most of the dispersive valves in dams are the Howell-Bunger type. However, Hollow-Jet valves remain in some dams, e.g., Entremontes Dam, Pentecoste Dam, and Passagem das Traíras Dam (ANA 2016). Technical reports produced by the same reference show that only in the northeast 
region of Brazil, there are at least 26 dams where dispersive valves can be found. According to the Brazilian Dams Safety Report of 2019, the situation of 156 dams in the country is critical (ANA 2020). Also, a report produced by SIMA (2019) synthetizes 202 dams constructed in the state of São Paulo, which were submitted to the National Dam Safety Policy. This major concern has substantially increased since the last survey, especially after the Brumadinho tragedy in 2019. In this context, special attention should be paid concerning cavitation in the Hollow-Jet valves to guarantee the operation of these dispersive valves.

CFD techniques are nowadays widely used to identify the behavior of cavitation in valves. Amirante et al. (2014) evaluated the effects of cavitation on the performance of a proportional directional valve. Nzombo (2017) analyzed the possibility of obtaining optimized projects of ball and butterfly valves, in which the cavitation potential could be minimized. Liu et al. (2019) studied the cavitation in diaphragm valves considering different openings. Tabrizi et al. (2014) analyzed the cavitation in ball valves using pressure drop and vortex formation for different openings. Lee et al. (2016) studied the optimization of part of a globe valve geometry to minimize the effects of cavitation. Wu et al. (2003) performed a threedimensional computational fluid dynamics study of cavitation in the Hollow-Jet valve used in the experiments of Wang (1999). They applied pressured-based algorithms, with the turbulence closure achieved by the $\kappa-\varepsilon$ turbulence equations, combined with a cavitation model, to simulate the turbulent cavitation flows. They found cavitation occurrence in the valve tip of their geometry. For all these works of literature, the common point, when performing the simulations, is the use of turbulence and cavitation models in order to solve the set of fundamental equations.

In this paper, following the previous work of Xavier et al. (2019), different types of two-equation turbulence models ( $\kappa-\varepsilon$ realizable, standard $\kappa-\omega$ and SST $\kappa-\omega)$ and cavitation models (Schnerr-Sauer, Singhal et al. and Zwart-Gerber-Belamri) were assessed considering a fixed operating point. The simulations were carried out with the academic software Ansys Fluent, and they were validated by a reproduction attempt of the results from $\mathrm{Wu}$ et al. (2003), and by comparison with the experimental results of Wang (1999). Economical solutions, as protective coatings made of specific materials and refurbishment processes, are also proposed in order to avoid damages caused by cavitation and the replacement of the valves.

\section{METHODOLOGY}

\subsection{Case of Study}

The original Hollow-Jet geometry $(\mathrm{d}=2.286 \mathrm{~m})$ was taken from the work of Morassi (2016). This valve is part of the dissipating chamber of Falcon Dam studied in that work and it is shown in Fig. 1 a). Before performing the simulations, some adaptations were necessary to reach the final geometry shown in Fig. 1 b).

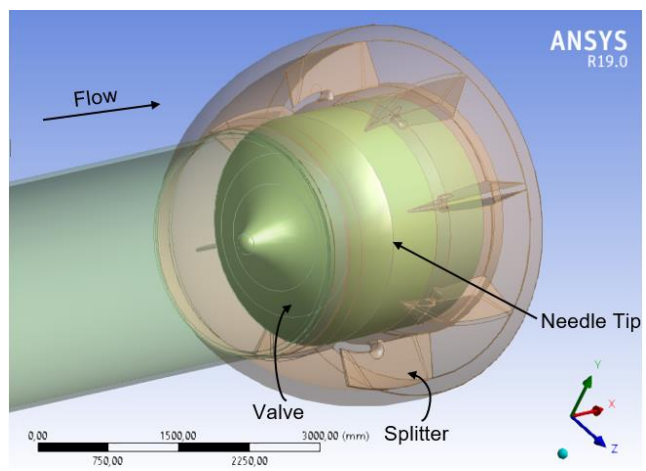

(a) original geometry

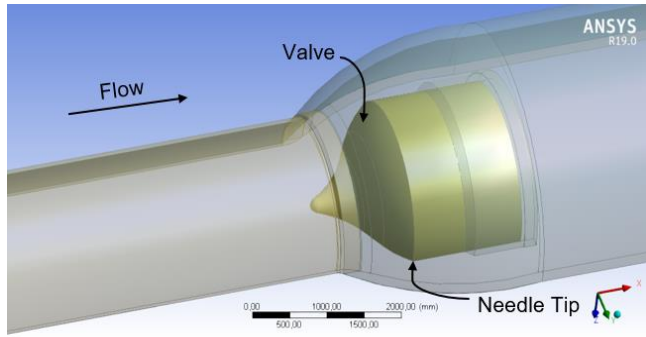

(b) adapted geometry

Fig. 1. Hollow-Jet geometry (100\% valve opening).

The boundary conditions to perform the simulations are described for each domain as follows:

a) Inlet: The most robust boundary conditions in problems with one inlet and one outlet are velocity inlet and pressure outlet. Besides this, for the present case the inlet pressure was unknown, therefore, velocity inlet was set as the boundary condition, in which a uniform velocity profile was set based on each flowrate considered. Before reaching the valve body, this velocity profile was already fully developed;

b) Outlet: According to Versteeg and Malalasekera (2007), if outlet boundaries are placed near to obstacles, it is possible that the flow has not yet reached a fully developed state and this may lead to significant errors. Based on that, and starting from the end of the original geometry, an extension of 10 times the valve's diameter was considered. For this boundary condition, a pressure outlet was selected and a zero static pressure was set, since the outlet is open to the atmosphere;

c) Symmetry: To decrease the simulation time without loss in accuracy and the quality of results, only the upper part of the geometry was taken to perform the simulations. To use this simplification, flow and flux through the symmetry plan could not exist, and normal velocities must be equal to zero; and

d) Wall: All the surfaces in contact with fluid are considered a wall. In this case, it is mandatory to have the no-slip condition as a boundary condition. Regarding the splitters which support the valve 
structure, they were removed for the simulations. According to Wang (1999) and Wu et al. (2003), these elements have a negligible thickness, and their shape does not modify the flow field.

Concerning grid configuration, the traditional tetrahedral mesh was initially set and modeled for the simulations. Nevertheless, thanks to the functionality of the software Ansys, this type of mesh was converted to polyhedral mesh, by forming polygons around each node in the tetrahedral mesh.

A great advantage of the polyhedral elements is that they have many neighbors, so the gradients can be better approximated when compared to the tetrahedral elements. Besides this, the computation time in that type of mesh is less than one-tenth of the time required by a tetrahedral mesh with the same accuracy. This happens because it is necessary approximately four times fewer elements and half of memory that would be used (PERIC; FEGURSON, 2005).

The final mesh was obtained by converting the tetrahedral mesh into a polyhedral mesh. For the boundary layer mesh, prism elements (characterized by having very high geometric anisotropy) were used through the inflation feature of the software Ansys. Due to the curvilinear shapes of the geometry, the smooth transition option of inflation was set, considering five layers, with the first grid cells in the range of $30<y^{+}<300$. In addition, a high Reynolds number approach with a wall function was also applied.

Moreover, a convergence and dependency study of the grid was performed, considering a residual error below $10^{-4}$ as stop criterion, resulting in a $3 \mathrm{D}$ mesh with 1.2 million elements. More characteristics about the final mesh can be verified in Xavier et al. (2019).

For the present work, a flowrate, equal to 64.76 $\mathrm{m}^{3} / \mathrm{s}$, was set as the operating point for the geometry showed in Fig. 1. b), which was completely open.

Due to the transitory nature of the cavitation phenomenon, all simulations were carried out considering unsteady flows.

The time step size used in this work was $\Delta t=$ $0.0001 s$, the same as Wang (1999) mentioned in his work. The smaller the time step size is, the more accurate the solution will be. However, the simulations time will be longer. Thus, the first 50 time steps considered this value to detect cavitation. The following time steps were performed, considering $\Delta t=0.005 \mathrm{~s}$.

The analysis of cavitation evolution was performed considering instant measurements throughout the flow. The dimensionless time $\left(\mathrm{t}^{*}=\mathrm{t} / 0.06\right)$, which physically represents the flow time, was used to follow the flow evolution, where the numerator represents the time duration of flow and the denominator is an arbitrary value with time dimension, chosen in order to adjust the values for the dimensionless time in the instant measurements studied.
The parameters analyzed in this paper are especially the contours of liquid volume fraction and the streamlines.

\subsection{Turbulence Closure Models}

Two-equation turbulence closure models are the most applied to close the set of fundamental equations. These models have the advantage of taking less processing time and an easy convergence process when compared to more elaborate models.

Besides this, the results, in general, have good accuracy and quality. In this paper, three turbulence models were described, and the results obtained from their use were compared.

\subsection{1 $\kappa-\varepsilon$ Realizable Model}

The $\kappa-\varepsilon$ models are the simplest and the most complete class of turbulence closure models of two equations. The focus here is the mechanism that affects the turbulent kinetic energy of the flow. Regular applications include heat transfer, combustion, and multiphase flow problems. According to Ansys Fluent (2013), initial studies have shown that the realizable model provides the best performance of all the model versions for several validations of separated flows, and flows with complex secondary flow features. In this work, for the simulations with the $\kappa-\varepsilon$ realizable model, the wall law was also selected.

\subsubsection{Standard $\kappa-\omega$ Model}

The standard $\kappa-\omega$ model was developed to model interactions near the wall more precisely than the $\kappa$ $\varepsilon$ models. However, the model can overestimate the shear stresses of the adverse pressure-gradient boundary layers, and it has problems with freestream flows. Also, this model is overly sensitive to the inlet boundary conditions, which is a disadvantage that had not been found in $\kappa-\varepsilon$ models. In general, it has been used for simulations on turbo machines and where strong vortices are present, such as on the tips of the wings.

\subsubsection{SST к- $\omega$ Model}

The SST $\kappa-\omega$ model is more popular in the aeronautics field because it is an improvement on the standard $\kappa-\omega$. This model can predict the separation and junction of flows in a better way than the $\kappa-\varepsilon$ and standard $\kappa-\omega$ models. In contrast, the model addresses some specific limitations of the base model, such as the sensitivity to freestream turbulence levels.

\subsection{Cavitation Models}

During the evaporation process and bubbles generation, an interface that separates vapor from the liquid is generated. In this interface, the fundamental conservation equations must be satisfied.

The phenomenon of growth and collapse of a single bubble cavity in a liquid could be described according to the general equation known as the 
Rayleight-Plesset equation. Cavitation models are developed based on this essential equation.

The software Ansys Fluent provides three cavitation models in its library. For the simulations in this paper, the models were assessed together with the turbulence closure models.

Moreover, for all models, zero slip velocity between the fluid and bubbles has been considered. According to Ansys Fluent (2013) and Şenocak (2002), it is always preferable to simulate cavitation using the mixture model without slip velocity.

\subsubsection{Zwart-Gerber-Belamri Model}

The Zwart-Gerber-Belamri (2004) model is different from the other models because it assumes that the bubble radius is constant for all bubbles in the liquid. It is also a robust model to predict cavitation with a good convergence.

On the other hand, the contribution of noncondensable gases was not considered. During the simulations, convergence was not achieved using this model.

\subsubsection{Singhal et al. Model}

The Singhal et al. (2001) model is a more complex model than the others. In the general equation, this model considers the effects of turbulence and noncondensable gases.

However, this model is overly sensitive to initial conditions, and it is numerically less stable and more difficult to use. Just like the Zwart-GerberBelamri model, it was not possible to achieve convergence during the simulations.

\subsubsection{Schnerr-Sauer Model}

The Schnerr-Sauer (2001) model uses an expression to connect the volume fraction of the vapor with the number of bubbles per volume of the liquid, and the radius of the bubble.

It is a robust model to predict cavitation, and convergence is usually easy. Just like the ZwartGerber-Belamri model, the contribution of noncondensable gases was not considered here.

Among the cavitation models available in the software and tested, this was unique where convergence was achieved and, therefore, it was set for the simulations with the different turbulence closure models.

\section{RESULTS AND DISCUSSION}

\subsection{Liquid Volume Fraction}

This parameter is very efficient for detecting cavitation in a system qualitatively and quantitatively. For the simulations performed, the contours of liquid volume fraction in the plane of a splitter were able to display the exact location of cavitation occurrence in the geometry.

The values assumed for the parameter go from 0 to 1 , where 0 indicates the presence of vapor only, and
1 indicates that there is only liquid. Any value inside this range indicates that vapor and liquid coexist.

\subsubsection{Liquid Volume Fraction and $\kappa-\varepsilon$ Realizable Model}

The contours evolution of liquid volume fraction for the $\kappa-\varepsilon$ realizable model is shown in Fig. 2.

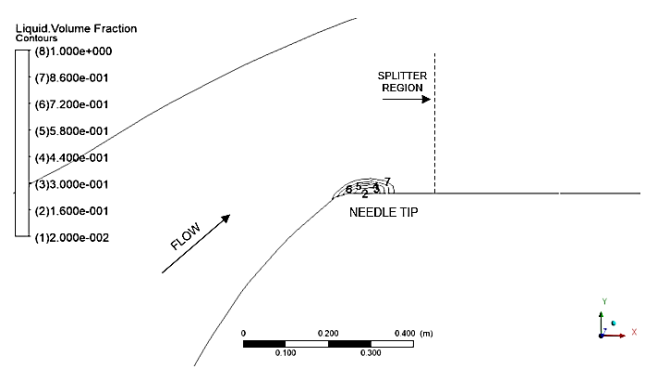

(a) $t^{*}=0.25$

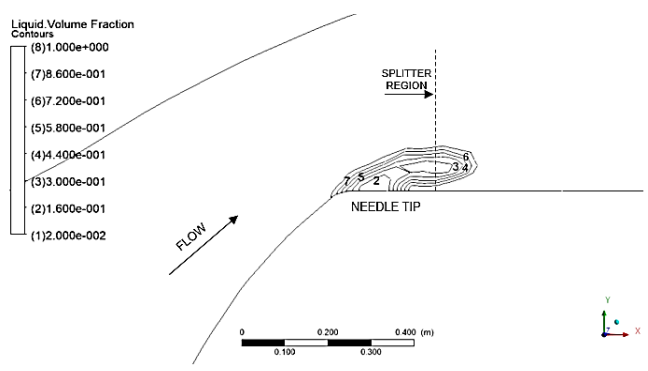

(b) $t^{*}=0.75$

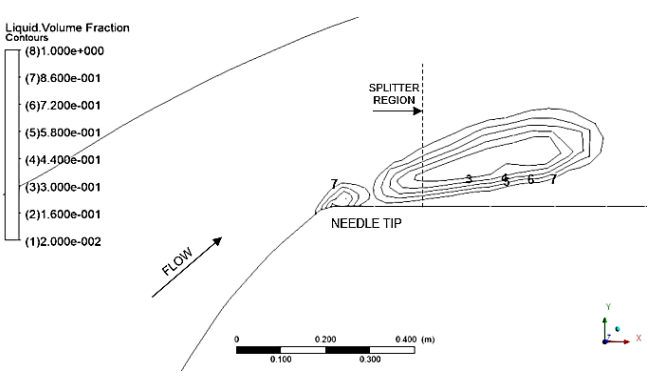

(c) $t^{*}=1.75$

Fig. 2. Contours of liquid volume fraction for the $\kappa-\varepsilon$ realizable model.

According to the results, when $t^{*}=0.25$, it is already possible to detect the formation of a cavity at the valve tip.

This cavity is stratified as a consequence of the values assumed by the liquid volume fraction and its size growths as long as the time flow increases. The cavity formation is due to the smallest pressures located in this region of the geometry.

At $t^{*}=0.75$, one can see that this cavity is already in contact with the splitter of the valve. Finally, when $t^{*}=1.75$, there is a detachment of the cavity by the tip.

For higher values of $t^{*}$, there was no new growth and detachment of the cavity. 
T. C. L. Xavier and J. P. Ortiz / JAFM, Vol. 14, No. 5, pp. 1399-1410, 2021.

\subsubsection{Liquid Volume Fraction and Standard $\kappa-\omega$ Model}

The contours evolution of the liquid volume fraction for this model is shown in Fig. 3.

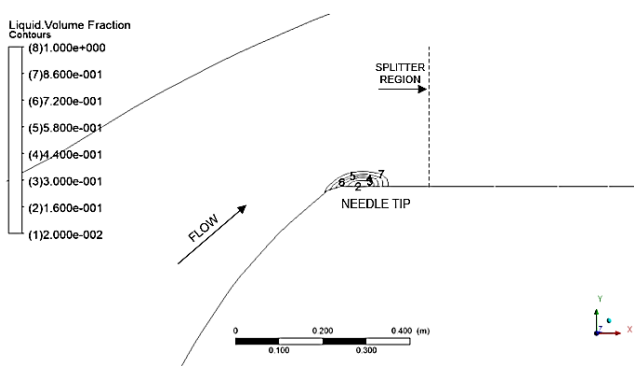

(a) $t^{*}=0.25$

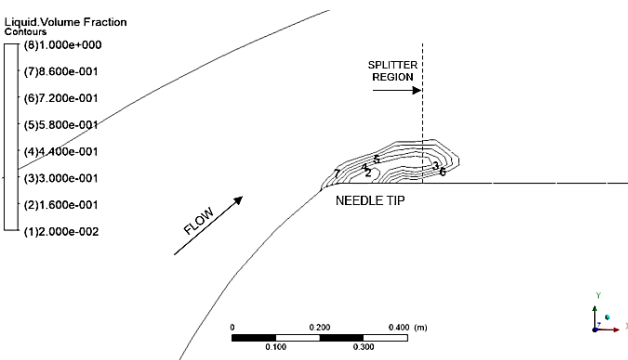

(b) $\mathrm{t}^{*}=0.75$

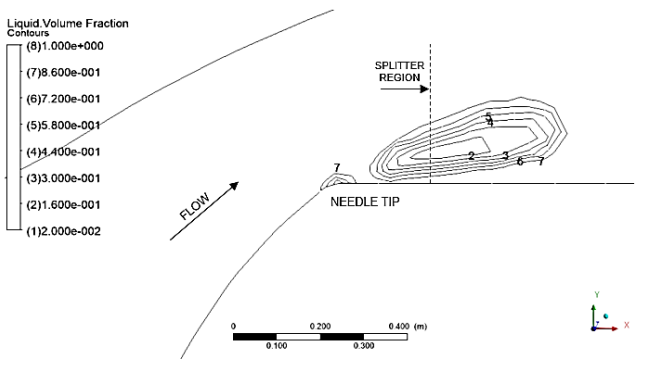

(c) $t^{*}=1.75$

Fig. 3. Contours of liquid volume fraction for the standard $\kappa-\omega$ model.

As for the previous case, one can also see the formation of a cavity at the valve tip at $t^{*}=0.25$. The process of growth and detachment at $t^{*}=1.75$ is again verified. There are no significant differences here compared to previous results. The main differences concern the values assumed for the parameter within the cavity, the arrangement of the contours, and the size of the main and the remaining cavity. For the remaining cavity at the tip, which has not detached, one can see that its size is smaller than the remaining cavity obtained in the $\kappa-\varepsilon$ realizable model. For the main cavity, the use of this model has generated a less elongated cavity, which may be a consequence of the streamlines and vortex associated.

\subsubsection{Liquid Volume Fraction and SST к- $\omega$ Model}

The contours evolution of liquid volume fraction for the SST $\kappa-\omega$ model is shown in Fig. 4. As for the previous cases, there is a formation of a cavity since $t^{*}=0.25$ with its growth and detachment at $t^{*}=$ 1.75. Again, slight differences can be verified when comparing the values assumed for the parameter inside the cavity, and the arrangement of contours. The dimensions of the main cavity detached are more similar to those of the $\kappa-\varepsilon$ realizable model but less elongated. One can also see that the size of the remaining cavity is more similar to the size obtained in the standard $\kappa-\omega$ model.

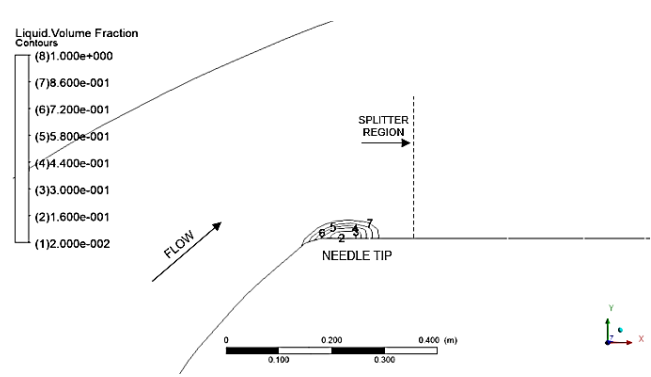

(a) $t^{*}=0.25$

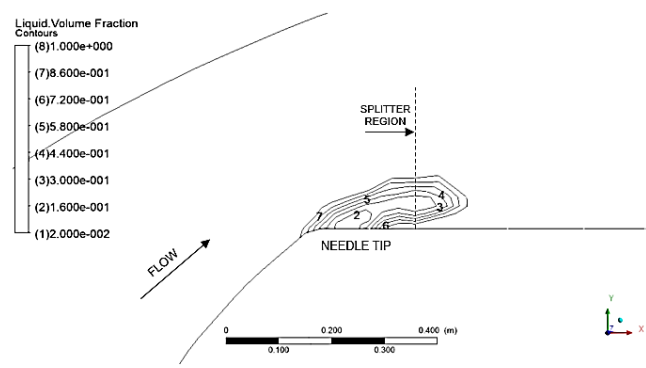

(b) $t^{*}=0.75$

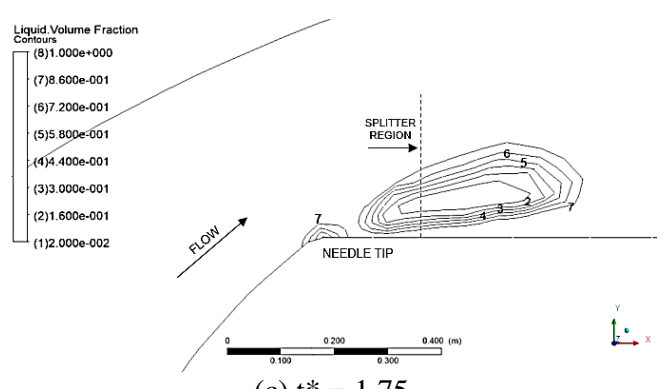

(c) $t^{*}=1.75$

Fig. 4. Contours of liquid volume fraction for the

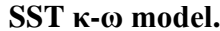

\subsection{Streamlines}

The streamlines are curves that are instantaneously tangent to the velocity vectors of the flow. These curves show the instantaneous massless particle movement, according to the Eulerian method of flow visualization. Therefore, the draw of streamlines is like an instantaneous photograph of the flow. They are extremely useful to detect vortex generation in a fluid, especially when referring to cavitation, where the vortex cavitation process may occur.

\subsubsection{Streamlines and $\kappa-\varepsilon$ Realizable Model}

Considering the same instants for the flow, Fig. 5 shows the distribution of streamlines for the $\kappa-\varepsilon$ realizable model. One can note that in the first 
instant at $t^{*}=0.25$, there is no signal of a vortex when the liquid passes through the geometry. When $t^{*}=0.75$, the streamlines are already starting to sketch a vortex formation. But at $t^{*}=1.75$, the vortex generation (just behind the valve tip) is very clear. The vortex generated is a cavitation vortex type, and it is the result of high-velocity gradients, mainly in the shear stresses region of the discharge pipe wall. When $t^{*}=1.75$, it is followed by the detachment and displacement of a cavity, as mentioned before.

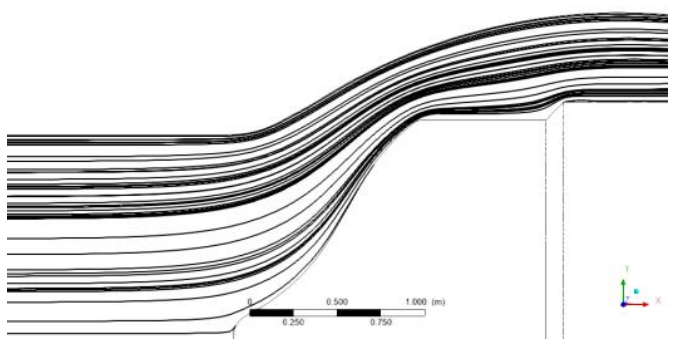

(a) $t^{*}=0.25$

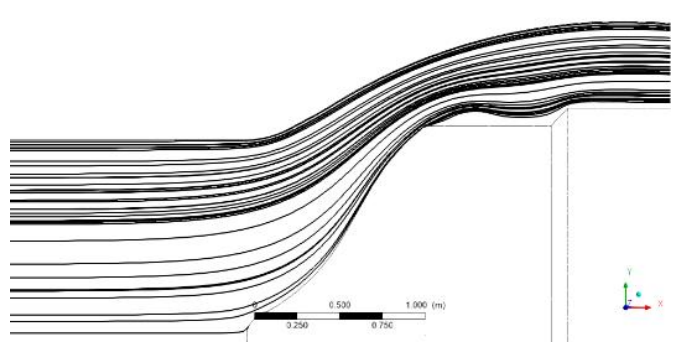

(b) $t^{*}=0.75$

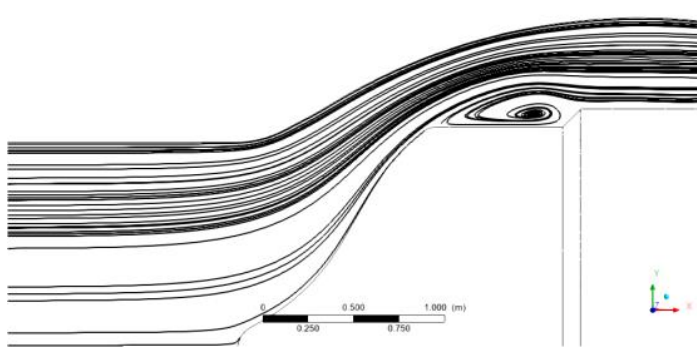

(c) $\mathrm{t}^{*}=1.75$

Fig. 5. Streamlines distribution for the $\kappa-\varepsilon$ realizable model.

\subsubsection{Streamlines and Standard $\kappa-\omega$ Model}

The distribution of streamlines considering the standard $\kappa-\omega$ model is shown in Fig. 6. Here, the same process of vortex generation is verified. Initially, when $t^{*}=0.25$, the streamlines show that there is no vortex formation. As the flow continues, at $t^{*}=0.75$, one can see that the lines start to deform. Finally, at $t^{*}=1.75$, the vortex is complete, and it is located just behind the valve tip, near the geometry surface. For the simulation with this turbulence model, some differences were detected in the shape of the vortex. The vortex generated in the results of the previous model is more developed, concentrating more streamlines in its interior when compared to the vortex obtained using the current model. It means that the capability of detecting a vortex is better using the $\kappa-\varepsilon$ realizable model.

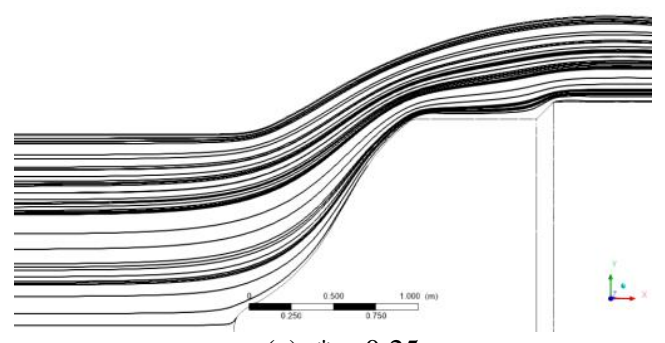

(a) $t^{*}=0.25$

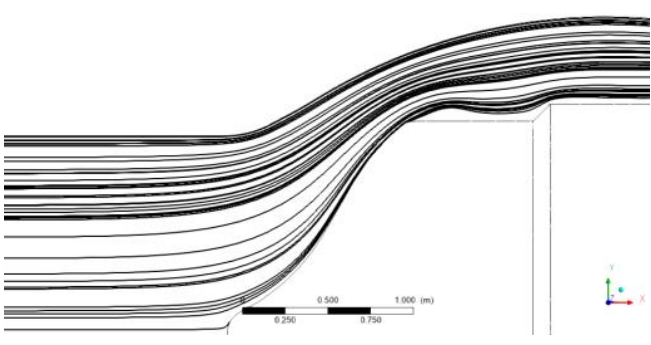

(b) $t^{*}=0.75$

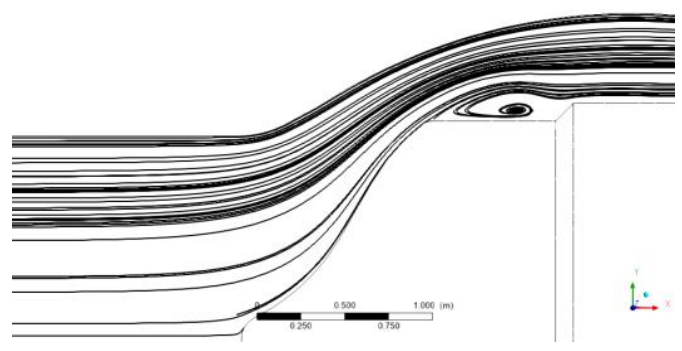

(c) $\mathrm{t}^{*}=1.75$

Fig. 6. Streamlines distribution for the standard

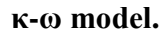

\subsubsection{Streamlines and SST $\kappa-\omega$ Model}

The distribution of streamlines considering the SST $\kappa-\omega$ model is shown in Fig. 7. Here, from $t^{*}=0.25$ to $t^{*}=0.75$, the results are quite similar to the results obtained for the other models. Again, the main differences concern the shape and concentration of streamlines inside the vortex when $\mathrm{t}^{*}=1.75$. Even though the SST $\kappa-\omega$ model is an improvement of the standard $\kappa-\omega$ model, no difference could be seen when the two models were compared.

\subsection{Validation of Simulations}

In order to try the validation of the results obtained in the simulations performed, the same methods and models applied in this work, preliminarly used in Xavier et al. (2019), were considered to reproduce the solutions of Wu et al. (2003), which are based on the experimental results of Wang (1999). Table 1 shows a comparison between the main methods and models, and Table 2 specifies the main parameters 
from Wu et al. (2003), also used in the attempt of validation.

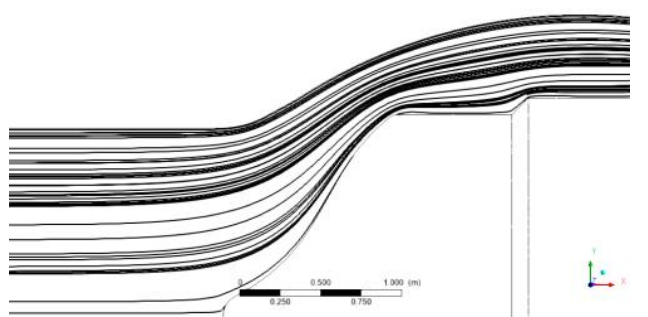

(a) $t^{*}=0.25$

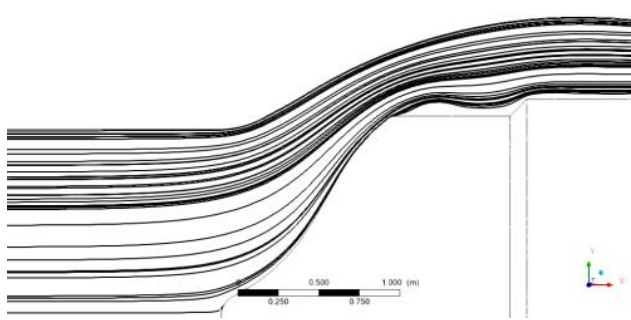

(b) $t^{*}=0.75$

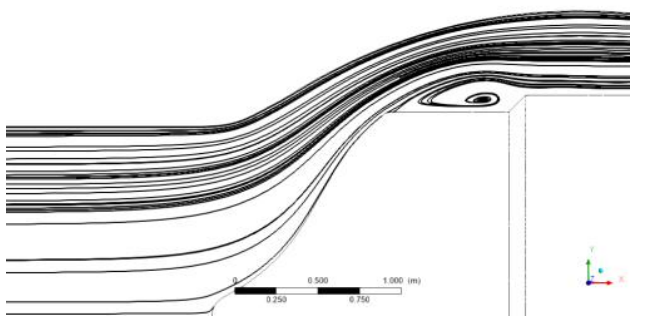

(c) $t^{*}=1.75$

Fig. 7. Streamlines distribution for the SST $\kappa-\omega$ model.

Table 1 Comparison of main methods and models.

\begin{tabular}{|c|c|c|}
\hline & $\begin{array}{c}\text { Current work/ } \\
\text { Xavier et al. } \\
\text { (2019) }\end{array}$ & $\begin{array}{l}\text { Wu et al. } \\
\text { (2003) }\end{array}$ \\
\hline $\begin{array}{l}\text { Solution of } \\
\text { unsteady } \\
\text { flow }\end{array}$ & PISO algorithm & $\begin{array}{c}\text { PISO } \\
\text { algorithm }\end{array}$ \\
\hline $\begin{array}{c}\text { Turbulence } \\
\text { model }\end{array}$ & RANS & FANS \\
\hline $\begin{array}{c}\text { Turbulence } \\
\text { closure } \\
\text { model }\end{array}$ & $\begin{array}{c}\kappa-\epsilon \text { realizable } \\
\text { with wall } \\
\text { functions }\end{array}$ & $\begin{array}{c}\text { standard } \kappa \\
\epsilon \text { with wall } \\
\text { functions }\end{array}$ \\
\hline $\begin{array}{c}\text { Cavitation } \\
\text { model }\end{array}$ & $\begin{array}{c}\text { Schnerr-Sauer } \\
(2001)\end{array}$ & $\begin{array}{c}\text { Kunz et al. } \\
(2000)\end{array}$ \\
\hline
\end{tabular}

Table 2 Main parameters for simulation.

\begin{tabular}{|c|c|c|}
\hline Parameter & Dimension & Unit \\
\hline Valve diameter & 0.812 & $\mathrm{~m}$ \\
\hline $\begin{array}{c}\text { Ratio between liquid } \\
\text { and vapor densities }\end{array}$ & 1000 & - \\
\hline Valve opening & $33 \%$ & - \\
\hline Reynolds number & 500000 & - \\
\hline Cavitation number & 0.9 & - \\
\hline
\end{tabular}

\subsubsection{Geometry and Grid configuration}

The geometry of Wu et al. (2003) was reproduced, and it is shown in Fig. 8.

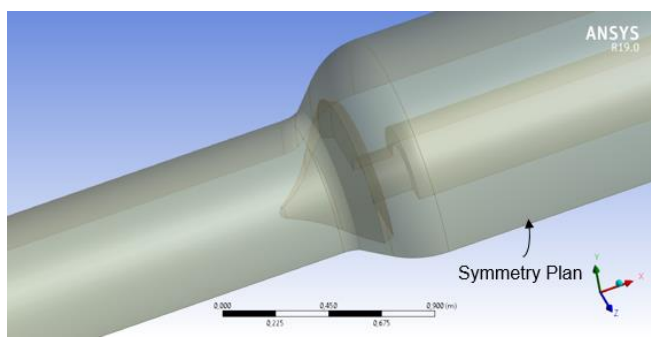

Fig. 8. Reproduction of geometry used in $\mathrm{Wu}$ et al. (2003).

A polyhedral mesh was applied to the geometry, considering the grid configuration, instead of a structured curvilinear one, as described in the work of literature. The mesh applied is shown in Fig. 9, where Fig. 9 a) shows the 3D mesh used in the simulations. Figure 9 c) shows the zoom of the mesh around the valve tip, delimited by the red rectangle in Fig. 9 b).

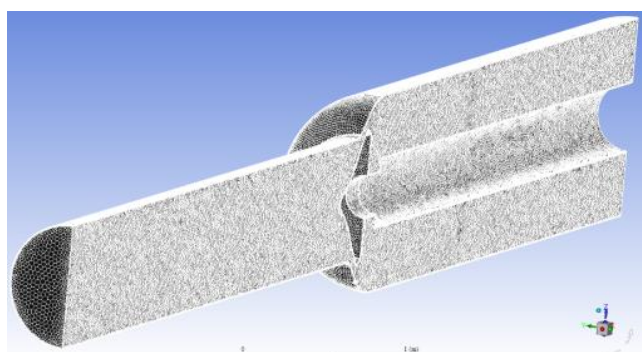

(a) 3D polyhedral mesh

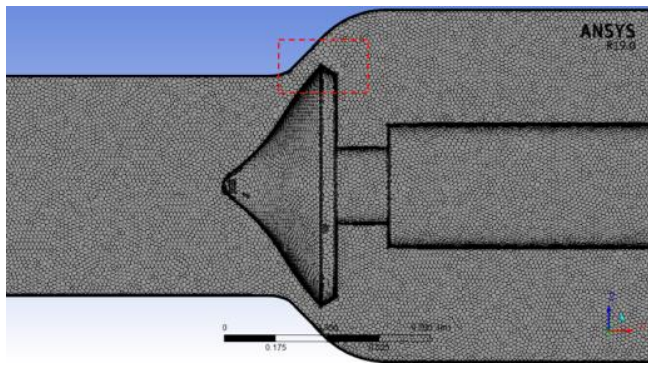

(b) symmetry plan of polyhedral mesh

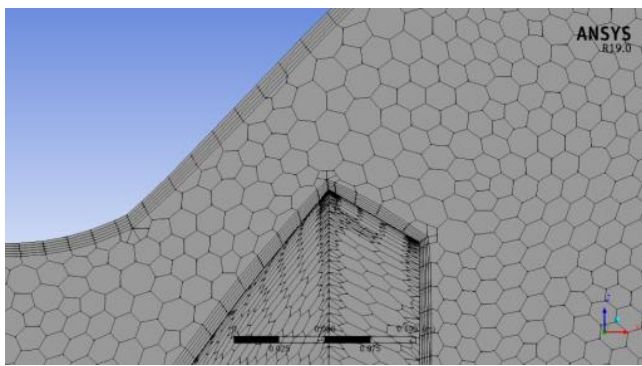

(c) mesh zoom at the valve tip region

Fig. 9. Polyhedral mesh for simulations.

As in the case of the study previously presented, inflation with five prisms layers was used and the first grid cells were also in the range of $30<y^{+}<300$. 
Before the simulation, a mesh convergence and dependence study was carried out. In order to perform this study, it was considered the first time step of $0.0001 \mathrm{~s}$, following Wang (1999), and a residual error below $10^{-4}$ as stop criterion. Finally, the average pressure at a point of the valve tip was obtained. The parameters are shown in Table 3.

Table 3 Mesh convergence and dependence characteristics.

\begin{tabular}{|c|c|c|c|}
\hline $\begin{array}{c}\text { Number of } \\
\text { tetrahedral/p } \\
\text { olyhedral } \\
\text { elements } \\
\text { (Millions) }\end{array}$ & $\begin{array}{c}\text { Grid points } \\
\text { for } \\
\text { polyhedral } \\
\text { mesh } \\
\text { (Millions) }\end{array}$ & $\begin{array}{c}\text { Gauge } \\
\text { Pressure } \\
(\mathrm{Pa})\end{array}$ & $\begin{array}{c}\Delta \\
(\%)\end{array}$ \\
\hline $3.0 / 0.6$ & 3.1 & 656945 & - \\
\hline $3.6 / 0.8$ & 3.7 & 647900 & 1.40 \\
\hline $4.5 / 1.0$ & 4.5 & 595993 & 8.71 \\
\hline $5.1 / 1.2$ & 5.1 & 600884 & 0.81 \\
\hline
\end{tabular}

In the first column of Table 3, one can see for each cell the number of tetrahedral elements and the corresponding number of polyhedral elements after the mesh conversion functionality, as previously explained. In the second column, the number of grid points for each polyhedral mesh after the conversion process is shown.

Based on the results in the third column and analyzing the fourth column, the polyhedral mesh with 1.0 million elements was selected, due to the fact that the gauge pressure measured for a finer mesh with 1.2 million elements resulted in a difference of gauge pressures less than the chosen criterion of $1 \%$. Finer meshes beyond 1.2 million elements have shown little differences in results.

\subsubsection{Comparison of Results}

The streamlines obtained are presented in Fig. 10. As one can verify, in both simulations and the experiment of the literature, there is a cavitation vortex formation. This vortex is located in the plane of a splitter. As previously explained, the vortex is a consequence of high shear stresses on the walls.

Comparing Fig. 10 a) and Fig. 10 b), one can see from the streamlines that the vortices are quantitatively different between them. Nevertheless, qualitatively speaking, there was a good agreement, specially regarding their location, which is in the same region of the geometry, and their shape.

This qualitative result is also in accordance with the result from the experiment showed in Fig. $10 \mathrm{c}$ ).

The contours of liquid volume fraction were also compared. They are presented in Fig. 11.

It is evident from Fig. 11 that a cavity with a mixture of vapor and liquid is present at the valve tip of both simulations and in the experiment. In this region, the smallest pressures of the geometry are perceived. The main difference in the results presented for the simulations consists of the fact that, in the simulation of this paper, showed in Fig. $11 \mathrm{~b}$ ), there was a detachment of the cavity from the valve tip. Which fact has not been proven in the simulation of the literature, showed in Fig. 11 a). Nevertheless, as shown in Fig. $11 \mathrm{c}$ ), in the experiment performed, the detachment of cavities from the valve tip was also verified.

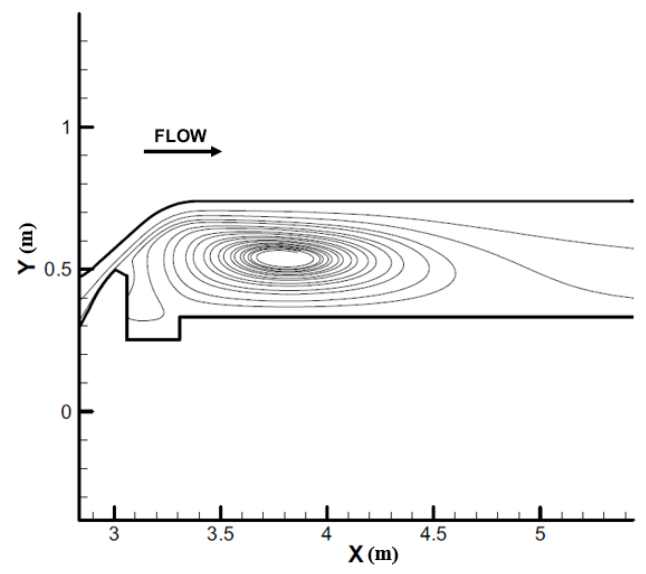

(a) streamlines of Wu et al. (2003)

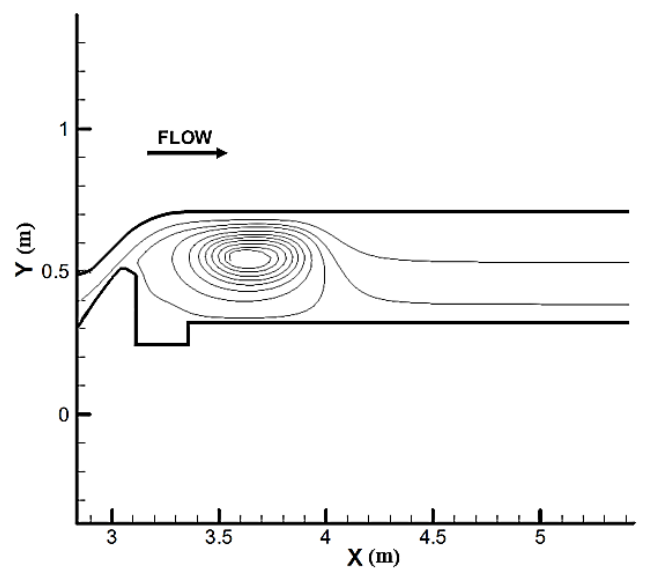

(b) streamlines obtained

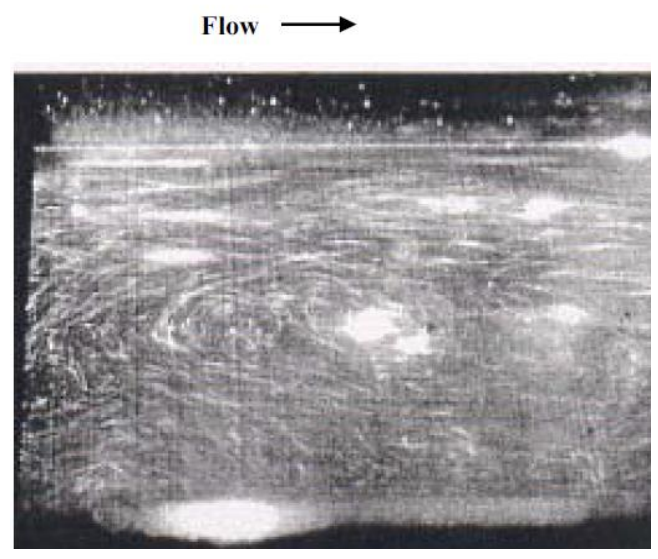

(c) flow pattern $(\mathrm{x}=3.4 \sim 4.2 \mathrm{~m})$

(Wu et al., 2003)

Fig. 10. Comparison of streamlines. 


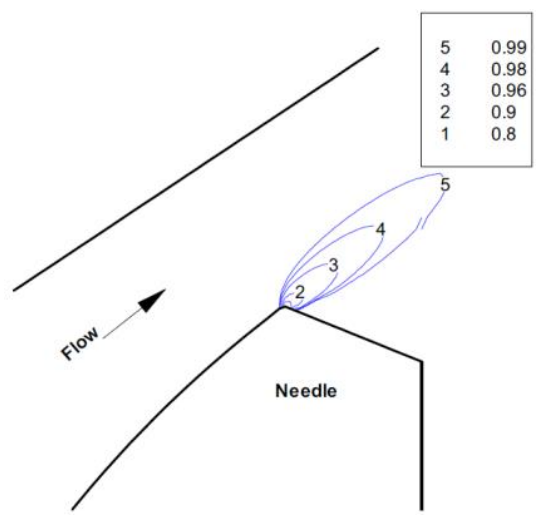

(a) contours of Wu et al. (2003)

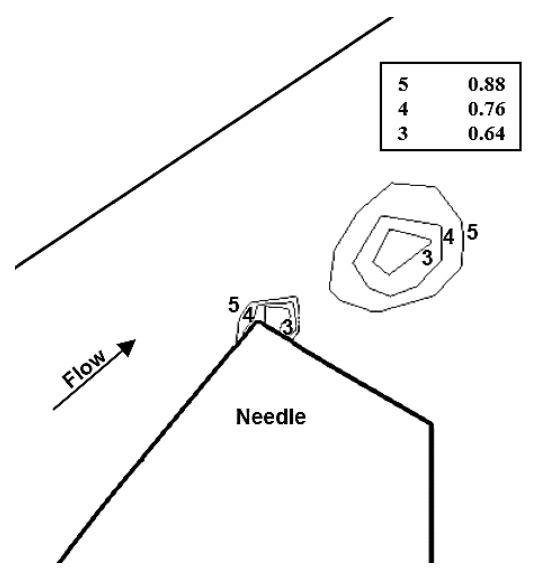

(b) contours obtained

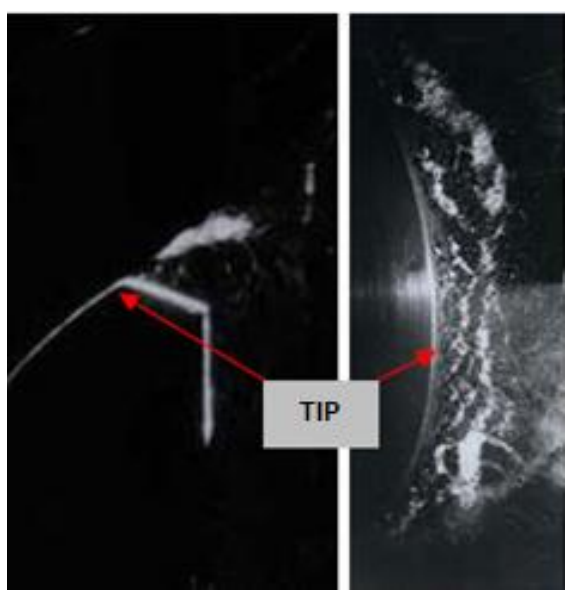

(c) cavity at needle tip (Wu et al., 2003)

Fig. 11. Comparison of liquid volume fraction

\subsection{Solutions to Cavitation in Hollow-Jet Valves}

To avoid or reduce cavitation in Hollow-Jet valves and their pipes, some mechanisms can be adopted, according to Tullis (1989):

a) Restrict the operation of the system to low levels of cavitation in such a way that the cavities do not contain much energy, and the collapse occurs before reaching the surface; b) Remove the surfaces of the cavitation area using a sudden enlargement, which should dissipate energy while avoiding cavitation;

c) Introduce air into the separation region to supersaturate the critical zone with air. It reduces the volumetric compressibility module of the liquid and dampens the collapse of the cavities by reducing the speed of the waves; and

d) Treat the surface with a material more resistant to erosion caused by cavitation. Typically, it is common to coat certain parts of the valves with stainless steel or other resistant material.

The solution proposed in a) implies changes in the operating points of the system. It could be a practical solution for systems containing the Hollow-Jet valves. Despite this, these systems and their dissipating chambers are designed to operate in predetermined ranges of flowrates. Furthermore, when the valve is completed open, the splitters are located close to the valve tip, so the collapse of cavities is probable. The solution in b) is not feasible, because it proposes modification in the original design of the valve. Solution c) is not the best choice to be adopted due to the amount of compressed air that needs to be injected into the valve, the feasibility of the solution, and also its costs.

Finally, considering recent developments concerning materials technology, the solution in d) seems to be the best, especially when regarding resistance to cavitation and cost-benefits associated.

\subsubsection{Coating Solutions on Hollow-Jet Valves}

The Hollow-Jet valve is the forerunner of the Howell-Bunger valve, which is currently the most commonly used free-discharge valve. Both share many of the same characteristics. The main difference is that the control of a Hollow-Jet valve is provided with an internal needle that moves upstream against the flow, while in the other type; the flow is controlled by the movement of the external sleeve, which seats against a fixed cone.

To prevent cavitation, $5 \%$ is the minimum opening of a Howell-Bunger valve, according to FEMA (2010). Beyond this value, in general, cavitation is not a big concern. This happens because this valve uses the principle of flow through an orifice discharging into an infinite enlargement. In this case, cavitation is expected to occur in the fluid, away from solid boundaries (Tullis 1989).

For the Hollow-Jet valve, cavitation is commonly present, especially around the splitters. Moreover, it is usually more expensive to fabricate them than the Howell-Bunger valve due to the complex shapes used in the needle portion and upstream body (FEMA 2010).

On the other hand, as explained in the beginning of this paper, many dams are refurbishing and reusing their Hollow-Jet valves to decrease costs and keep their original operation. 
In special, for the Hollow-Jet valves of Yellowtail Dam (EUA), a more sophisticated work was performed to recover and protect the component (Hydro Review 2009).

For the recovery, a complete work of refurbishment was necessary, in accordance with the steps described in Fairall et al. (1984).

For the protection, the old coatings of Yellowtail Dam, from 1988, were removed, and special coatings were applied to the valve's surfaces. These coatings were the FLEXICLAD ${ }^{\mathrm{TM}}$ DuraTouch DL in the most vulnerable areas to cavitation and the METALCLAD CeramAlloy ${ }^{\mathrm{TM}} \mathrm{CL}+$ for the other surfaces. Both products are from the company ENECON.

FLEXICLAD $^{\mathrm{TM}}$ DuraTouch DL is an elasticceramic polymer composite formed by two components, $100 \%$ solid. After mixed with its activator, the product acquires fluid consistency. It is specially formulated to protect the surfaces of equipment subject to accelerated cavitation and corrosion/erosion, due to its toughness and properties.

METALCLAD CeramAlloyTM CL+ is a highperformance polymer composite formed by two components, $100 \%$ solid. It is used for the repair/recovery of surfaces and its components have excellent resistance to erosion and corrosion. When mixed, the product also becomes a viscous liquid.

\subsubsection{Cost Estimates for Refurbishment and Coating}

The main advantage in coating the Hollow-Jet valve is the cost-benefit of this process, mainly because only the more susceptible parts to cavitation are required to be protected. The coated parts can be, therefore, limited to the sum of areas related to the splitters, the base of these splitters, and the valve body downstream of the tip.

To calculate the total costs of coating, the product FLEXICLAD $^{\mathrm{TM}}$ DuraTouch DL (recommended to cavitation and erosion/corrosion) was considered. Information about costs of the product was estimated based on discussions with its local supplier, ENECON Brazil.

The Hollow-Jet valve selected for this study is the same used for the main simulations. Table 4 shows the analysis of all costs estimated.

The economy we have is evident when a complete renovation process is executed instead of replacing the valve. According to the table, this economy is about $72 \%$ when compared to the purchase of a Howell-Bunger valve, which dimensions are not so different.

Besides, this solution can be adopted in Brazil, for example, where the criticality of many dams requires quick and economical solutions. In this case, the Hollow-Jet valve reform process adds all these benefits, ensuring the safety and operation of dams.
Regarding safety, Brazilian National Water Agency (ANA) is making all efforts to increase the database of the various dams around the country. Hence, more dams operating with the Hollow-Jet valves in different levels of conservation will be detected.

Table 4 Refurbishment and coating costs analysis.

\begin{tabular}{|c|c|c|}
\hline Parameter & Dimension & Unit \\
\hline Coated area & 30 & $\mathrm{~m}^{2}$ \\
\hline Coating cost & 24509 & $\mathrm{U} \$$ \\
\hline $\begin{array}{c}\text { Refurbishment } \\
\text { cost (Hydro } \\
\text { Review 2012) }\end{array}$ & 198285 & $\mathrm{U} \$$ \\
\hline Total cost & 222794 & $\mathrm{U} \$$ \\
\hline $\begin{array}{c}\text { Price of a } \\
\text { Howell-Bunger } \\
\text { valve type with } \\
\text { d }=2.743 \text { m } \\
\text { (USACE 2017) }\end{array}$ & 800000 & $\mathrm{U \$}$ \\
\hline $\begin{array}{c}\text { Ratio between } \\
\text { total cost for } \\
\text { refurbishment } \\
\text { and valve price }\end{array}$ & $27.8 \%$ & - \\
\hline
\end{tabular}

\section{CONClusion}

The cavitation phenomenon in the Hollow-Jet valves was detected during the simulations performed. The phenomenon may be considered a cavitation vortex class. Cavities were verified at the valve tip due to the smallest pressures in this region. In all simulations of this work, the detachment of the cavities was perceived in the direction of the flow. Their collapse with the splitters and other structures is the main consequence of cavitation damages.

The comparison of turbulence and cavitation models showed that the best couple was using the Schnerr-Sauer cavitation model with the $\kappa-\varepsilon$ realizable turbulence model. The $\kappa-\varepsilon$ realizable model was more sensitive to detect the vortex generation, and the contours of liquid volume fraction were quite similar to the contours of the other models. Regarding the cavitation models, convergence was only possible using the SchnerrSauer model.

The results presented here in an attempt to validate the simulations by reproducing and comparing results from the literature were relatively satisfactory. It was previously highlighted that different methods and models were considered, and some differences in the results were already expected. This is the case, for example, of the vortices reproduced, where a qualitative accuracy was possible. Moreover, some limitations existed due to difficulties in obtaining information from all steps followed by the comparison work. The most important thing here is to note that the physics of the problem has been reproduced, and the methods 
and models used in this work can be considered acceptable and validated.

The solutions to treat the cavitation in Hollow-Jet valves were also presented. Due to the original design of the valve, some solutions are not possible. In some countries, the solution consisting of refurbishment and covering the valve surface with coatings is being increasingly replicated. In fact, this is a good solution to avoid the disposal of this type of valve and because the replacement of a preexisting valve is not always possible due to design compatibility issues. Systems are initially designed considering a specific type of valve. The replacement of the component by another requires a complete dimensioning study to be feasible.

Moreover, costs are probably the main reason to keep the original valve. According to the cost estimates performed here, covering the valve is much more economical than replacing it, and the CFD simulations presented in this article are an important help to the decision-makers in the choice, replacement, or refurbishment of the valves.

Finally, this subject is especially important to Brazil, due to the high quantity of risky dams spread across the country, as mentioned at the beginning of this article, and especially after some tragedies in the last years, generating more concerns and increasing the number of inspections and regulations about the safety of dams.

\section{REFERENCES}

Amirante, R., E. Distaso and P. Tamburrano (2014). Experimental and Numerical Analysis of Cavitation in Hydraulic Proportional Directional Valves. Energy Conversion and Management 87, 208-219.

ANA (2016). Reservoirs in the Brazilian Semiarid Region: Hydrology, Hydric Balance and Operation, Engecorps Engenharia S.A., Summary Sheets, Brazil.

ANA (2020). Dams Safety Report 2019, Brasília, Brazil.

Ansys - Fluent User's Guide $15.0 \quad$ (2013, November). Pennsylvania, United States.

Fairall, R., H. Task, T. Simms and C. Quinton (1984, June). Rebuilding the Hollow-Jet Outlet Valves at Monticello Dam, Solano Project, Water Operation and Maintenance, Bulletin $\mathrm{N}^{\circ} 128$, United States.

FEMA (2010, June). Technical Manual: Outlet Works Energy Dissipation, P-679, EUA, 62.

Hydro Review (2009, May). Coatings Provide Smooth Surface on Hollow Jet Valves at Yellowtail, Waterpower XVI Conference and Exhibition, Issue 4, Volume 28, 32-34.

Hydro Review (2012, July). Reclamation Awards Contract for Anderson Ranch Dam Parts Refurbishment, North America, Rehabilitation and Repair, Turbines and
Mechanical Components, EUA.

Kakki-Anathodu, D. (2019, July). Operation and Maintenance Manual, Doc. No. 2_DSO_O\&M_SGHEP: Kakki \& Anathodu Dams_v1, Kerala State Electricity Board Ltd, Pallom, Kottayam, India.

Kunz, R. F., D. A. Boger and D. R. Stinebring, T. S. Chyczewski, J. W. Lindau, H. J. Gibeling, S. Venkateswaran and T. R. Govindan (2000). A Preconditioned Navier-Stokes Method for Two-Phase Flows with Application to Cavitation Prediction. Computers \& Fluids 29, 849-875.

Lee, M. G., C. S. Lim and S. H. Han (2016). Shape Design of the Bottom Plug Used in a 3-Way Reversing Valve to Minimize the Cavitation Effect, International Journal of Precision Engineering and Manufacturing 17(3), 401406.

Liu, Y., L. Lu and K. Zhu (2019, September). Numerical Analysis of the Diaphragm Valve Throttling Characteristics. Processes 7, 671.

MANANG (2015, March). Bissorte Dam Drain Valve Track, Hydroelectric Construction, Savoie, France.

Morassi, R. S. (2016). Study with CFD on the Operating Range of the Dissipation Chamber for Dispersive Valves. Master's Dissertation, University of São Paulo, São Paulo, Brazil.

Nzombo, D. N. (2017) A Numerical Investigation of Cavitation in Valves and Techno-Economic Analysis of Pinewood Solvent Liquefaction, Master of Science Thesis, Iowa State University, Graduate Theses and Dissertations (15593).

Peric, M. and S. Ferguson (2005). The Advantage of Polyhedral Meshes. CD-adapco, 1-2.

Schnerr, G. H. and J. Sauer (2001). Physical and Numerical Modeling of Unsteady Cavitation Dynamics. In Fourth International Conference on Multiphase Flow, New Orleans, USA.

Senocak, I. (2002). Computational Methodology for the Simulation of Turbulent Cavitating Flows, Ph.D. Dissertation, University of Florida, Gainesville, USA.

SIMA (2019). Dams in the State of São Paulo: Report of the Working Group, Secretariat for Infrastructure and Environment, São Paulo State Government, Brazil.

Singhal, A. K., H. Y. Li, M. M. Athavale and Y. Jiang (2001). Mathematical Basis and Validation of the Full Cavitation Model, ASME FEDSM'01, New Orleans, Louisiana.

Tabrizi, A. S., M. Asadi, G. Xie, G. Lorenzini and C. Biserni (2014) Computational FluidDynamics-Based Analysis of a Ball Valve Performance in the Presence of Cavitation, ISSN 1810-2328, Journal of Engineering 
T. C. L. Xavier and J. P. Ortiz / JAFM, Vol. 14, No. 5, pp. 1399-1410, 2021.

Termophysics 23(1), 27-38.

Tullis, J. P. (1989). Hydraulic of Pipelines: Pumps, Valves, Cavitation, Transients, John Wiley \& Sons, Inc., Utah State University Foundation $87,125-126$.

USACE (2017, June). Replacing a Fixed Cone Valve on Summersville Dam, Huntington District Public Affairs Staff.

USBR (2019). Budget Justifications and Performance Information, Fiscal Year 2019, 42-43.

USSD (2017). Improving Reliability of Commonly Used Hydraulic Valves, Denver, Chapter 2, 10.

Versteeg, H. K and W. Malalasekera (2007). An Introduction to Computational Fluid Dynamics - The Finite Volume Method. Pearson Prentice Hall, Second Edition, 280-
283.

Wang, G. (1999). A Study on Safety Assessment of a Hollow-Jet Valve Accompanied with Cavitation, Ph.D. Dissertation, Tohoku University, Sendai, Japan, 16-86.

Wu, J., I. Senocak, G. Wang, W. Wu and W. Shyy (2003). Three-Dimensional Simulation of Turbulent Cavitating Flows in a Hollow-Jet Valve. CMES 4(6), 679-689.

Xavier, T. C. L., R. S. Morassi and J. P. Ortiz (2019). CFD Modelling of Cavitation in Hollow-Jet Dispersive Valves, 38th IAHR World Congress, v. SS1, 904-913.

Zwart, P. J., A. G. Gerber and T. Belamri (2004). A Two-Phase Flow Model for Predicting Cavitation Dynamics, In Fifth International Conference on Multiphase Flow, Yokohama, Japan. 